\title{
The challenges of understanding animal minds
}

\author{
Jeffrey R. Stevens* \\ Center for Adaptive Behavior and Cognition, Max Planck Institute for Human Development, Berlin, Germany \\ *Correspondence: jstevens@mpib-berlin.mpg.de, jeffrey.r.stevens@gmail.com
}

Comparative psychology is by nature an interdisciplinary science that lies at the crossroads of psychology and biology but also draws from other fields in the natural, social, and cognitive sciences. The study of the psychology of animals has been labeled animal cognition, comparative cognition, animal learning, animal psychology, and animal intelligence. Here, comparative psychology is used interchangeably with these terms, encompassing all fields that explore the psychological mechanisms underlying animal behavior, including human behavior.

The primary goal of comparative psychology is to understand the cognitive, emotional, and motivational processes of the animal mind. How do other animals perceive, learn about, and make decisions in their worlds? From our pets to exotic animals portrayed in nature documentaries, we are inherently curious about other animals. Comparative psychology both provides a window into their minds, as well as offers a unique perspective on the human mind. Which aspects of our psychology do animals share? Human uniqueness is constantly challenged as we learn more about the psychology of animal minds. Once distinctive human abilities - such as tool use, language, and mental time travel - appear, at least to a degree, in other species. Though other species exhibit elements of these abilities, the central question for comparing humans and animals remains, do humans and other animals share the same psychological mechanisms?

Comparative psychology explores many of the same topics as human psychology. From learning and memory to communication and decision making, the field investigates a number of key questions, for example:

- How do animals understand causal relationships in their environments?

- Can animals represent the perceptions, intentions, and beliefs of others?

- Do animals plan for the future?
- Can animals use referential communication?

- How do animals track time and number?

- Do animals maintain a cognitive map of their environment?

- Do animals attend to the well being of others?

- How do animals categorize objects in their world?

- Which emotional and motivational factors underly animal behavior?

Studying the psychology of other species is not easy. With rare exceptions, we cannot ask directly about their psychological states. Instead, we must make inferences about the psychology of animals based on their behavior. Inferring internal mechanisms from external behavior results in a number of challenges for comparative psychologists. Though we have met a number of these challenges, several remain, slowing our progress in advancing comparative psychology. To push forward, we must meet these conceptual and practical challenges head on.

\section{THE COMPLEXITY OF PARSIMONY}

The difficulties of making inferences about internal mechanisms has spawned two general approaches to studying cognitive aspects of animal behavior. The animal learning approach emphasizes the general learning principles, such as instrumental, and Pavlovian conditioning, espoused by Hull, Spence, Tolman, and Skinner. The cognitive approach examines other forms of cognition such as perception, attention, memory, categorization, navigation, timing, number, communication, decision making, and social cognition. Though learning mechanisms often are considered simpler explanations, some cognitive mechanisms are more complex, requiring an organism to generate a mental representation. Since Morgan's (1903) admonition to opt for the most parsimonious explanation supported by the data, the learning tradition has set the benchmark for parsimonious explanations. If a simpler, learning account can explain the data, then we should not invoke a more complex explanation. This stringent benchmark has set a high bar for researchers in the cognitive tradition to reject simple learning explanations. Occasionally, the bar is raised higher and higher, sometimes to the extent that the learning mechanism becomes quite complex. How complex does a parsimonious explanation need to be before we accept a representational one? How do we define the complexity of a mechanism? One solution is to sidestep the parsimony/complexity question completely. Rather than resorting to a scale of psychological complexity, we can develop and test more precise models of the phenomena of interest.

Even after excluding simpler explanations for phenomena, the question arises, under what circumstances do animals use the more complex form of cognition? Possessing a particular psychological ability does not imply using it at every opportunity. In many cases, animals may use simpler mechanisms, reserving the more complex mechanisms for situations in which the simpler mechanisms do not work. The circumstances under which animals use different mechanisms remains an open question in comparative psychology.

\section{INDIVIDUAL DIFFERENCES}

A hallmark of data in comparative psychology is variation across individuals. Despite this, we have very little understanding of the sources of variation in psychological mechanisms. Evolutionary models predict individual differences in behavior (Wolf et al., 2007), but few models explore why cognition and emotion should vary across individuals. A number of questions arise when developing a theoretical understanding of individual differences. Is there really variation in animal psychological abilities or only in the expression of these abilities? To what extent is this variation adaptive? To what extent does the variation result from environmental influences? Are there general 
reasons for variation in psychology or do we need to consider each ability separately? Researchers of animal behavior investigate individual differences under the headings of animal personality, behavioral syndromes, and temperament. We must now extend this study of variation at the psychological level and explore the sources of such variation.

\section{PUTTING THE COMPARATIVE BACK INTO COMPARATIVE PSYCHOLOGY}

Despite the name and origins of the field, comparative psychology lacked many comparative studies during much of the twentieth century. Only a handful of species acted as the workhorses of the field: the pigeon, the rat, and the rhesus macaque. Focusing on a few species offers a more in-depth and cumulative method for understanding the psychology of those species. This restriction, however, limits our broader understanding of psychological mechanisms in animals. Comparative psychologists have lamented this lack of diversity for decades (Beach, 1950; Cook, 1993; Shettleworth, 1993), and, more recently, researchers have tested a number of interesting questions in a wide variety of species, ranging from insects and octopods to tortoises and manatees. We should continue to meet the challenge of casting a wide taxonomic net.

Cataloging the abilities of various species provides a necessary first step in understanding the psychology of animals. However, comparative does not mean simply collecting information on a broad range of species; it also implies active comparison across species (Shettleworth, 1993). The implicit comparison in the early days of comparative psychology was between a single animal species and humans. Comparisons have become more explicit recently, borrowing methods directly from fields such as cognitive psychology, developmental psychology, social psychology, and economics. When testing questions of human psychology in animals, we should strive to make the experiments as comparable as possible across species.

Comparing not just to humans but across animal species opens a host of new questions to investigation. In particular, the comparative method allows the testing of evolutionary questions regarding the origins of and ecological pressures on psychological mechanisms. Do phylogenetically closely related species share similar psychological mechanisms? What kinds of evolutionary pressures shape psychological mechanisms across species? Does the social environment play a special role in shaping psychology? How can we test whether species share actual mechanisms rather than just general psychological abilities? Comparisons across species pose difficulties and require great care regarding the species tested and the experimental methods used. Nevertheless, the fruits of this labor will yield valuable insights into the nature of animal psychology.

\section{ECOLOGICAL RELEVANCE}

Simon (1990) argued that a serious study of cognition must explore both the mind and the environment in which the mind interacts. He likened this to two blades in a pair of scissors that cannot function unless both blades are present. This provides an important lesson for comparative psychology as well. Studies of comparative psychology frequently face criticism for overly artificial task environments. Using simple stimuli in impoverished apparatuses offers clear advantages in terms of reducing the likelihood of confounding variables. Unfortunately, this gain in internal validity comes at the price of reducing external validity. Striking a balance between careful control over the experimental situation and eliciting meaningful responses is critical to understanding animal psychology (Cook, 1993). The right balance starts with asking ecologically relevant questions. What kinds of mechanisms do animals need to navigate through their physical and social worlds?

The next step is to develop experimental stimuli and tasks that tap the natural abilities of animals. Though simple artificial stimuli offer complete control over the features presented to animal subjects, Cook (1993) argues that more naturalistic stimuli of intermediate complexity can offer a reasonable balance between control and ecological relevance. In addition, Hare (2001) argues that we must consider the natural ecology of the species to develop appropriate experimental tasks. When placed in unnatural situations, animals may not demonstrate the relevant psychological mechanism. Though not easy to conduct, field experiments can offer the most naturalistic circumstances for testing psychological mechanisms in animals. Developing tasks that tap natural behaviors and situations can yield more valid studies of comparative psychology.

\section{STRONG INFERENCE}

Much of comparative psychology is based on existence proofs: Do animals have theory of mind, metacognition, episodic memory, empathy, or other-regarding preferences? To further mature, however, the field needs to develop more sophisticated models of how psychological mechanisms work, not just whether they are present and what influences them. Weisman (2008) offers an important challenge to the young researchers in behavioral and cognitive science: embrace Platt's (1964) notion of strong inference. Strong inference emphasizes the development of multiple hypotheses and the design of critical experiments allowing for alternative outcomes that will exclude some of the hypotheses. Currently, many behavioral scientists rely on testing a single hypothesis against a null hypothesis, despite the pitfalls of this approach (Marewski and Olsson, 2009). Rather than only testing for the presence or absence of a psychological ability or for factors that influence an ability, we need to explore how the processes work. To this end, testing a series of formal process-based models allows a precise understanding of psychological mechanisms. Though formal models are common in the animal learning tradition (e.g., models of timing), other areas of comparative psychology have yet to use these models to their full potential.

Developing and testing multiple hypotheses yields more careful inferences because more powerful statistical techniques can be used. Instead of relying on the weak null hypothesis testing paradigm, we can use competitive model testing techniques to discriminate between hypotheses. Incorporating process models and competitive model selection into the strong inference approach can only raise the level of rigor for comparative psychology.

\section{REPLICATION AND REPRODUCIBLE RESEARCH}

Around the turn of the twentieth century, a horse named Clever Hans captivated first Germany and then the world with amazing feats of arithmetic skill. Alas, the even more clever Pfungst (1911) debunked the horse by demonstrating that Hans simply used 
inadvertent cues from his owner or others during the questioning. This story is often the first cautionary tale learned by all comparative psychologists as they enter the field. It reminds us that the wishes and biases of the observer can influence the observation. It also has encouraged meticulous methodologies for researching animal psychology. Our field could improve, however, in fostering replication and reproducible research.

A core principle of experimental research methods is to replicate studies. This is common practice in human psychology experiments, perhaps because a new pool of participants is available each semester at the university. The typically small sample sizes used in comparative psychology can make replication difficult. Nevertheless, we should encourage the general practice of replicating our work and publishing these replications, even if they result in different findings. As one solution to facilitating replications, we can grant free access to experimental methods. The Comparative Mind Database ${ }^{1}$ offers a systematic attempt to maintain free access to experimental methods in comparative psychology by archiving experimental protocols and video clips of experimental sessions. This database facilitates comparative studies by standardizing experimental procedures such that researchers may use similar protocols across species. In addition to exact replications of methods, we need to replicate our studies using different methods. As scientists, we often become enamored with our methodologies. Yet, the use of a single method to test a hypothesis can result in a biased view of the phenomenon of interest. Returning to Simon's scissors, cognition cannot be understood fully without accounting for the environment, and ensuring that our findings generalize across multiple experimental environments is crucial. Replicating our work within laboratories, between laboratories, and across methodologies strengthens the integrity of our conclusions.

${ }^{1}$ http://www.cmdbase.org/
Allowing free access to data and data analyses is equally important in advancing comparative psychology because it allows for reproducible research (de Leeuw, 2001). With the available electronic technologies, scientists can include data files either as supplementary materials published on a journal's website or posted on the author's website. In addition to publishing the data, granting access to how the data were analyzed can be useful for other scientists. Some statistical software programs, including the freely available $\mathrm{R}$ software for statistical computing ${ }^{2}$, allow the reporting of not only statistical results but also the code used to generate the results. Making this code freely available facilitates both error checking and direct reproduction of data analysis. Thus, posting experimental protocols, example videos, data files, and analysis code maintains the transparent nature of science. In the spirit of the mission of open access, we at Frontiers in Comparative Psychology encourage the publication of methods, data, and data analysis along with scientific articles.

\section{LOOKING AHEAD}

Comparative psychology explores the fascinating topic of the animal mind. The field has made great strides in the recent years, though more work remains. We face exciting conceptual challenges such as grappling with issues of parsimony and complexity, exploring individual differences, and posing careful comparative questions of psychological mechanism. We also share more practical challenges with other areas in behavioral science and beyond, including developing more naturalistic experimental paradigms, implementing strong inference techniques, and producing reproducible and transparent research. No single piece of scientific work will likely address all of these challenges. Nevertheless, to advance comparative psychology into the twentyfirst century, we must carefully craft our

${ }^{2}$ http://www.r-project.org/ questions and continually be mindful of the most rigorous means to achieve our goal of delving into the minds of animals.

\section{ACKNOWLEDGMENTS}

I wish to thank Lael Schooler, Sara Shettleworth, and Alison Stevens for comments on this paper.

\section{REFERENCES}

Beach, F.A. (1950). The Snark was a Boojum. Am. Psychol. 5, 115-124.

Cook, R. G. (1993). The experimental analysis of cognition in animals. Psychol. Sci. 4, 174-178. doi: 10.1111/ j.1467-9280.1993.tb00483.x.

de Leeuw, J. (2001). Reproducible Research: The Bottom Line. Department of Statistics Papers, University of California, Los Angeles.

Hare, B. (2001). Can competitive paradigms increase the validity of experiments on primate social cognition? Anim. Cogn. 4, 269-280. doi: 10.1007/s100710100084.

Marewski, J. N., and Olsson, H. (2009). Beyond the null ritual: formal modeling of psychological processes. J. Psychol. 217, 49-60. doi: 10.1027/00443409.217.1.49.

Morgan, C. L. (1903). Introduction to Comparative Psychology, 2nd Edn. London: Walter Scott.

Pfungst, O. (1911). Clever Hans: (The Horse of Mr. Von Osten.) A Contribution to Experimental Animal and Human Psychology. New York: Henry Holt.

Platt, J. R. (1964). Strong inference. Science 146, 347-353.

Shettleworth, S. J. (1993). Where is the comparison in comparative cognition? Alternative research programs. Psychol. Sci. 4, 179-184. doi: 10.1111/j.14679280.1993.tb00484.x.

Simon, H. A. (1990). Invariants of human behavior. Annu. Rev. Psychol. 41, 1-20. doi: 10.1146/annurev. ps.41.020190.000245.

Weisman, R. G. (2008). Advice to young behavioral and cognitive scientists. Behav. Processes 77, 142-148. doi: 10.1016/j.beproc.2007.09.004.

Wolf, M., van Doorn, G. S., Leimar, O., and Weissing, F. J. (2007). Life-history trade-offs favour the evolution of animal personalities. Nature 447, 581-584. doi: 10.1038/nature05835.

Received: 15 September 2010; accepted: 28 October 2010; published online: 19 November 2010.

Citation: Stevens JR (2010) The challenges of understanding animal minds. Front. Psychology 1:203. doi: 10.3389/ fpsyg.2010.00203

This article was submitted to Frontiers in Comparative Psychology, a specialty of Frontiers in Psychology.

Copyright (c) 2010 Stevens. This is an open-access article subject to an exclusive license agreement between the authors and the Frontiers Research Foundation, which permits unrestricted use, distribution, and reproduction in any medium, provided the original authors and source are credited. 